\title{
Um modelo ubíquo para retriagem de pacientes baseado na Internet das coisas
}

\author{
Guilherme Wunsch ${ }^{1}$, Cristiano André da Costa ${ }^{1}$, Rodrigo da Rosa Righi ${ }^{1}$ \\ ${ }^{1}$ Programa de Pós-Graduação em Computação Aplicada \\ Universidade do Vale do Rio dos Sinos (UNISINOS) \\ emaileguiwunsch.com, \{cac,rrrighi\}@unisinos.br
}

\begin{abstract}
Triage is a process which aims to sort patients according to their need for care in emergency department. Within this process there is re-triage, which occurs when another assessment is performed if the patient has clinical needs for it or on a fixed period defined by triage protocols. This study aimed to develop a computer model which use the concepts of mobile and ubiquitous computing focused on healthcare in patients re-triage stage. The main scientific contribution of this study was infer the correct re-triage moment collecting the vital signs of the patients with the use of Internet-of-things. For the evaluation of the proposed model was developed a prototype trying to analyze its application in a created scenario. Thus, we concluded that the developed model is viable to use in emergency department.
\end{abstract}

Resumo. Triagem é um processo que visa ordenar os pacientes de acordo com sua necessidade de cuidados em emergências de hospitais. Contida nesse processo existe a retriagem, que ocorre quando uma outra avaliação é efetuada caso haja necessidades clínicas para isso ou em um período fixo e recorrente definido pelo protocolo do triagem. Esse trabalho se propôs a desenvolver um modelo computacional que visa utilizar conceitos de computação móvel e ubíqua focados nos cuidados de saúde durante a etapa de retriagem de pacientes. A principal contribuição científica desse trabalho foi inferir o momento correto para efetuar a retriagem através da coleta de sinais vitais dos pacientes fazendo o uso da Internet das coisas. Para a avaliação do modelo proposto foi desenvolvido um protótipo buscando analisar sua aplicação em um cenário criado. Sendo assim, nós concluímos que o modelo desenvolvido é viável para uso nas emergências de hospitais.

\section{Introdução}

Triagem é um processo realizado em hospitais para classificar os pacientes de acordo com sua necessidade de cuidados [Jentsch et al. 2013]. Atualmente, protocolos robustos de triagem vem sendo amplamente utilizados nos hospitais do mundo todo [Christ et al. 2010]. A retriagem é uma etapa importante contida nesse processo e ocorre quando uma nova avaliação do paciente é efetuada caso haja necessidades clínicas para isso [Rahmat et al. 2013]. Segundo [Christ et al. 2010], três dos quatro principais protocolos de triagem - Australasian Triage Scale (ATS), Canadian Triage And Acuity Scale (CTAS), Manchester Triage System (MTS) e Emergency Severity Index (ESI) - inserem 
a retriagem em seu fluxo. Sendo assim, para essas etapas serem bem sucedidas, necessitamos de profissionais treinados e artefatos que auxiliem e proporcionem maior agilidade e precisão, tornando este processo efetivo e funcional.

Em apenas um dos principais protocolos de triagem é definida uma periodicidade para a retriagem dos pacientes. Entretanto, os demais protocolos, que aplicam essa etapa durante o processo de triagem, apenas adotam que a retriagem deve ser utilizada quando necessário [Christ et al. 2010]. Sendo assim, por um lado, pode-se estar fazendo verificações desnecessárias junto aos pacientes caso adotada uma periodicidade fixa para efetuar a retriagem. Por outro lado, aplicá-la quando necessário é, de certa forma, muito subjetivo. Nesse aspecto é importante conseguir determinar o momento correto da retriagem visando eliminar esses problemas mencionados.

A Computação ubíqua (ou computação pervasiva) é usada para descrever a onipresença dos computadores no cotidiano das pessoas [Weiser 1991]. Assim, tem se desenvolvido aplicações cada vez mais adaptáveis, capazes de interpretar um contexto e antecipar futuras ações que devem ser efetuadas [Schilit et al. 1994, Marilza Pernas et al. 2012]. Em função disso, esse trabalho se propõe a estudar a computação ubíqua com o objetivo de propor um modelo computacional para auxiliar os enfermeiros na retriagem de pacientes em emergências de hospitais usando também a Internet das coisas afim de detectar o melhor momento para efetuar a retriagem.

Este artigo está estruturado nas seções a seguir. A segunda apresenta a fundamentação teórica utilizada. A terceira traz o modelo da solução proposta. Na sequência, a quarta seção apresenta a metodologia de avaliação. A quinta descreve os trabalhos relacionados. Por fim, a conclusão deste trabalho é apresentada, na sexta seção.

\section{Fundamentação teórica}

A ideia da tecnologia estar inserida de forma natural no cotidiano das pessoas foi visionada em 1991 por Weiser. O autor defende que o conceito, denominado computação ubíqua, tornaria a interação entre o ambiente, usuário e tecnologia invisíveis [Weiser 1991]. Segundo [Satyanarayanan 2011], esse conceito só pôde ser alcançado nos dias de hoje através de inovações na tecnologia de redes sem fio, maior eficiência energética e hardwares cada vez mais portáteis com softwares cada vez mais adaptáveis.

Essa adaptação de softwares pôde ser alcançada através do estudo de ciência de contexto. Conforme [Schilit et al. 1994], com a computação móvel os dispositivos estão constantemente trocando seu ambiente de execução. A quantidade de processadores disponíveis para determinada tarefa, dispositivos de entrada acessíveis para o usuário, capacidade de rede e custos de conexão podem mudar ao longo do tempo e lugar. Explorar essa troca é o objetivo da ciência de contexto [Schilit et al. 1994]. Ampliando esse conceito, [Marilza Pernas et al. 2012] define que, através de ciência de situação, é possível proporcionar que aplicativos tenham a capacidade de perceber e compreender a situação atual, com a finalidade de antecipar ou prever sua demanda em um futuro próximo.

Com a finalidade de antecipar essa demanda, pode-se fazer o uso de dispositivos inteligentes interconectados para auxiliar no entendimento de um determinado contexto. Isso vai ao encontro do uso de Internet das coisas. Esse conceito promete criar um mundo onde todos os objetos ao redor são conectados a Internet e se comunicam uns 
com os outros sem a necessidade de intervenção humana [Atzori et al. 2010]. Segundo [Blake 2015], a Internet das coisas para cuidados de saúde tem emergido recentemente como uma abordagem para cuidados de saúde personalizados.

Esse é o objetivo dos cuidados de saúde de forma ubíqua. Ou seja, aproximar os serviços médicos em ambos os lados, tanto para pacientes quanto para profissionais da saúde. Um sistema para cuidados de saúde de forma ubíqua implica em um ambiente que pacientes possam receber, a qualquer momento, tratamento, assim como, também, possam transmitir remotamente informações pertinentes à sua saúde. [Gelogo and Kim 2013].

\section{Modelo proposto}

A arquitetura geral do modelo proposto buscou integrar todos os conceitos definidos anteriormente em uma arquitetura de sistemas distribuídos, denominada Service-oriented architecture (SOA), ou arquitetura orientada à serviços. $\mathrm{O}$ principal fundamento da SOA é a disponibilização das funcionalidades de um sistema na forma de serviços [Krafzig et al. 2005]. Sendo assim, como observado na Figura 1, as regras de negócio e dados armazenados pelo modelo estão localizados nesse serviço, que será descrito posteriormente na Subseção 3.1. Por outro lado, os clientes, futuramente descritos na Subseção 3.2, são aplicativos distribuídos em dispositivos móveis que podem ser utilizados tanto pelos enfermeiros quanto pelos pacientes (enquanto aguardam o atendimento).

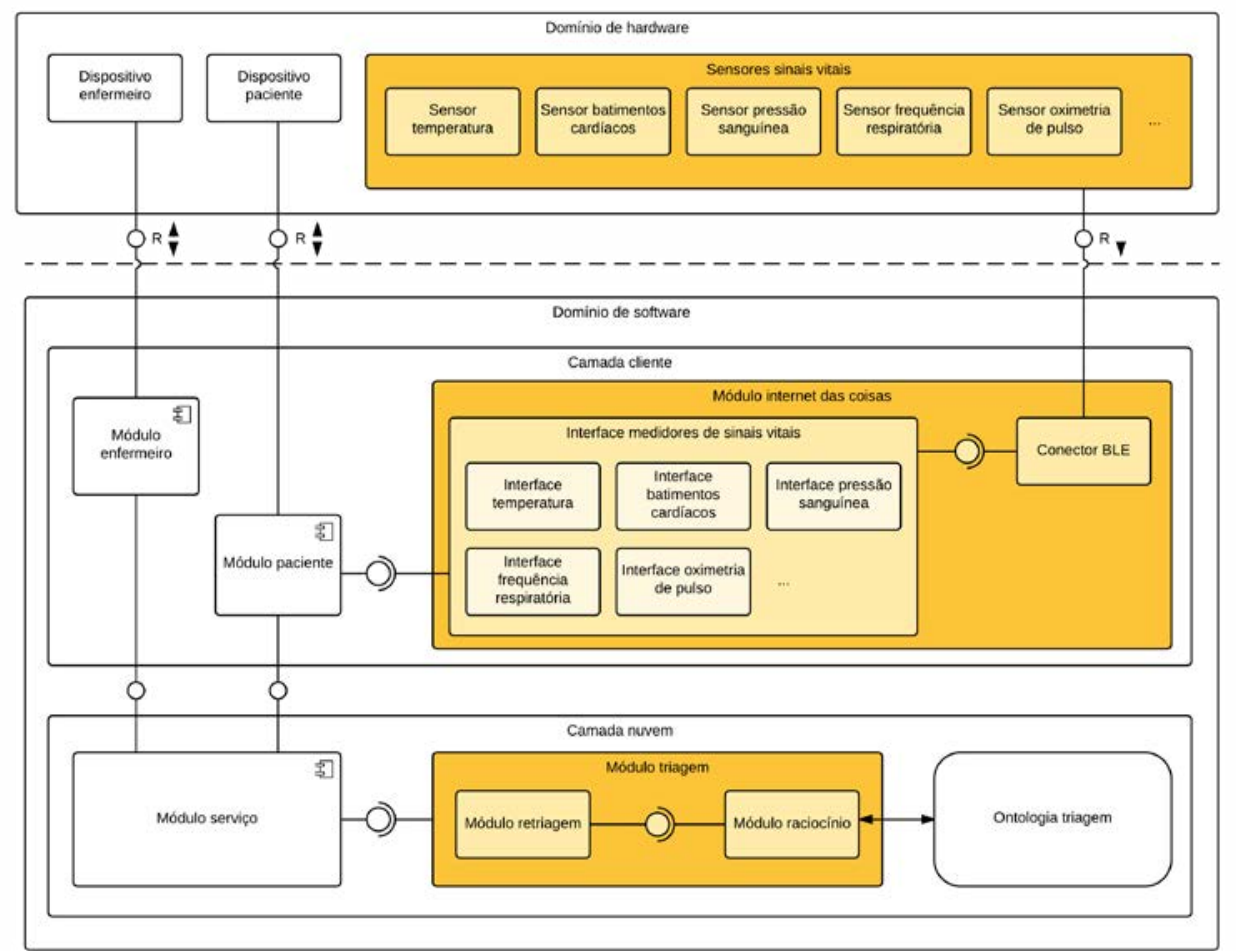

Figura 1. Visão geral do modelo proposto.

\subsection{Camada nuvem}

A camada nuvem é onde se localiza o serviço do modelo. Esse serviço disponibiliza uma interface REST para que todos os clientes possam acessá-la de forma fácil, rápida e eficaz. 
Nessa camada, as regras de negócio do modelo estão organizadas de forma hierárquica, tendo como principal objetivo prover informações voltadas ao processo de triagem a todos os clientes da solução. Nesse caso, ela é responsável por receber as informações coletadas diretamente dos pacientes através dos dispositivos vestíveis, alimentar um subsistema conectado a uma ontologia do protocolo de triagem utilizado e, através de uma técnica de raciocínio, inferir a triagem do paciente de acordo com os dados coletados. O módulo mais importante dessa camada é o responsável pela retriagem dos pacientes. Seu papel é interpretar todos os dados de sinais vitais coletados em tempo real, analisá-los e, novamente, através de raciocínio na ontologia do protocolo avaliar se houve alguma mudança na triagem do paciente para então disparar uma notificação ao enfermeiro responsável caso necessário. Essa ontologia pode ser personalizada de acordo com o protocolo de triagem utilizado pelo hospital. Um modelo dela pode ser observado na Figura 2.

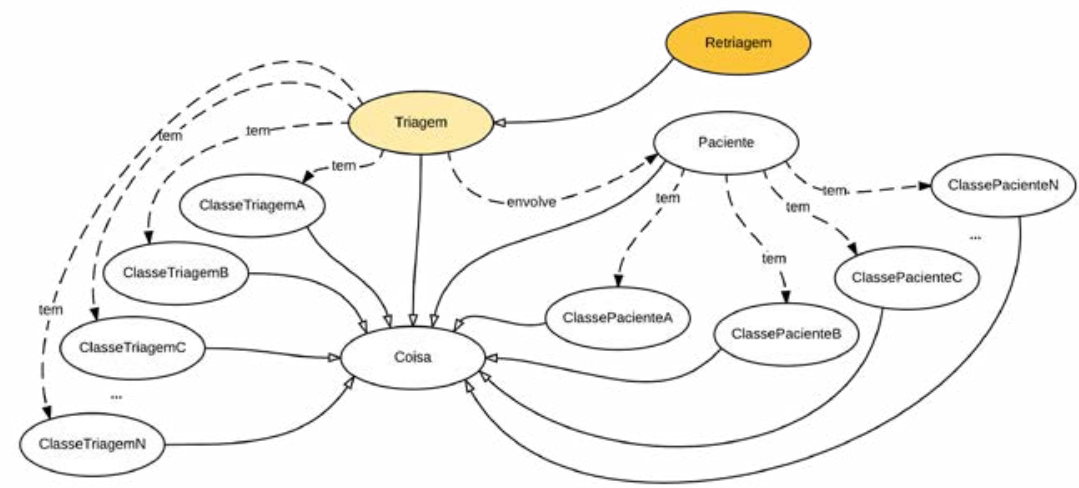

Figura 2. Modelo da ontologia para definir a triagem e retriagem do paciente.

Note que no diagrama desenvolvido na Figura 2, as classes da ontologia são representadas por figuras ovais. Setas sólidas representam a hierarquia entre classes e subclasses, enquanto setas pontilhadas são utilizadas para representar as propriedades entre as instâncias dessas classes. Os indivíduos dessa ontologia são, por exemplo, apresentações, discriminadores e sintomas dos pacientes que estão inseridos em suas respecitivas classes relacionadas à triagem (ClasseTriagemA, B, C, .. e e N). Eles também são, por exemplo, grupos de idade, gênero e demais informações relacionadas ao paciente através de suas respectivas classes (ClassePacienteA, B, C, ... e N). Os indivíduos dessa ontologia devem estar relacionados através de regras e propriedades de transitividade com as suas respectivas categorias de triagem para que um raciocinador consiga inferir a triagem de um paciente com base nas informações obtidas do paciente.

\subsection{Camada cliente}

A camada cliente proposta nesse modelo, no entanto, é constituída por aplicativos desenvolvidos para dispositivos móveis. Esses aplicativos devem auxiliar tanto enfermeiros como os pacientes durante o processo de triagem e enquanto o paciente está aguardando atendimento médico. No caso dos enfermeiros, o aplicativo auxilia em toda a gestão do fluxo de pacientes na emergência do hospital e agiliza o processo de coleta de dados na triagem. Esses dados vão desde informações pessoais até os sinais vitais, que podem ser obtidos de dispositivos vestíveis instalados diretamente nos pacientes através de uma ponte criada a partir do aplicativo do paciente. Além disso, os enfermeiros também 
podem receber notificações (disparadas pelo serviço da solução) quando for necessário efetuar a retriagem de algum paciente. Por outro lado, os pacientes dispõem apenas de informações voltadas ao seu processo de triagem, como, por exemplo, o tempo em fila, sua posição atual, estimativa de tempo para o atendimento, etc. Esse aplicativo do paciente também servirá como agregador dos dispositivos vestíveis instalados em seu corpo que visam monitorar seu estado de saúde através dos sinais vitais.

Esses dispositivos vestíveis, usados para aferir os sinais vitais dos pacientes, conectam-se com o aplicativo do paciente (no momento da triagem e enquanto aguarda pelo atendimento) através de redes de conectividade de baixo alcance como ZigBee, Bluetooth, Wireless Body Area Networks (WBAN), etc [Atzori et al. 2010]. O modelo se propõe a integrar apenas dispositivos compatíveis com Bluetooth Low Energy (BLE) visto que essa é uma das principais tecnologias no mercado para esses sensores vestíveis e é compatível também com smartphones, tablets e phablets que estão sendo vendidos. Sendo assim, um módulo importante da camada cliente é o de Internet das coisas. Esse módulo possui um conector BLE responsável por receber a comunicação vinda dos diversos dispositivos vestíveis conectados e modulariza-la, através de uma interface para cada tipo de sensor, de modo que o restante do sistema possa entender qual tipo de sinal vital esta sendo recebido em dado momento e em qual unidade de medida pré-definida ele deve ser interpretado. Esse módulo é uma peça fundamental, pois existem hoje no mercado muitos tipos de dispositivos dos mais diversos fabricantes.

\section{Avaliação}

A comunidade científica tem empregado o uso de cenários para avaliação de aplicações ubíquas e sistemas sensíveis ao contexto [Satyanarayanan 2011, da Rocha et al. 2015]. Segundo os autores [Westergren et al. 2014], com o uso dessa metodologia na área da saúde é possível tomar uma decisão mais consciente, pois eliminamos o fator humano e focamos apenas nos reais problemas envolvidos. Além disso, ela proporciona uma economia de custo e tempo e, também, não são envolvidas questões éticas como, por exemplo, aplicar dois processos de triagem ao mesmo paciente [Worster et al. 2007, Jobé et al. 2014]. Com o objetivo de avaliar o modelo descrito anteriormente, foi criado um cenário que descreve uma situação fictícia de uma paciente que chegou à emergência do hospital e foi devidamente triada. Após algum tempo na fila de espera, apresentou alterações significativas em seus sinais vitais e precisou ser retriada. A seguir é descrito o cenário utilizado para a avaliação do modelo proposto:

"Uma menina de dezesseis anos de idade, na qual foi enviada para casa, pois não estava se sentindo bem na escola, chegou com sua mãe na emergência. Ela é asmática há cinco anos. O hospital possui enfermeiros treinados e aptos a utilizar o protocolo de Manchester no processo de triagem. Durante a triagem, o enfermeiro verificou que a paciente está completamente lúcida e mencionou que tem estado cada vez mais ofegante a dois dias. Seus batimentos cardíacos e a saturação de oxigênio, aferidos no momento da triagem através de dispositivos vestíveis são respectivamente $100 \mathrm{bpm}$ e $92 \%$. O enfermeiro utilizou o fluxograma para asma do protocolo de Manchester e definiu a classificação amarela à paciente. A menina foi dirigida até a sala de espera ainda com os dispositivos vestiveis para o monitoramento, em tempo real, dos seus sinais vitais. Durante a espera sua pulsação sofreu uma alteração para 110 bpm e a saturação de oxigênio para $90 \%$. Automaticamente o sistema enviou uma notificação para o enfermeiro responsável pela 
triagem para que fosse efetuada a retriagem da paciente. Então, baseado nas alterações nos sinais vitais, a paciente foi reclassificada com a cor laranja, ganhando maior prioridade no atendimento de acordo com o protocolo aplicado pelo hospital."

Complementando a avaliação proposta para o modelo, foi desenvolvido um protótipo de aplicação, para iOS, que se comunica com um serviço na nuvem, também desenvolvido para esse processo. A elaboração do protótipo foi efetuada com base no modelo proposto e dividida em três etapas de desenvolvimento: criação do serviço, desenvolvimento do aplicativo para dispositivos móveis e desenvolvimento do módulo de integração com os sensores vestíveis que monitoram os sinais vitais do paciente.

O serviço foi desenvolvido utilizando a linguagem PHP em conjunto com a framework Symfony ${ }^{1}$. A camada de interface do serviço, responsável por disponibilizar os recursos que são acessados pelos aplicativos dos pacientes e enfermeiros, foi construída utilizando o padrão REST para transferir objetos JavaScript Object Notation (JSON) sobre o protocolo Hyper Text Transfer Protocol Secure (HTTPS). Já para a persistência dos dados foi utilizado o Sistema de Gerenciamento de Banco de Dados (SGBD) MySQL. Em conjunto com o banco de dados, foi utilizada a Application Programming Interface (API) de mapeamento objeto relacional para PHP denominada Doctrine ${ }^{2}$, que é distribuída junto com a framework Symfony. Esse serviço é responsável por gerenciar todo o processo de triagem, que é atribuída ao paciente através de um processo de raciocínio efetuado em uma ontologia, e, além disso, notificar aos enfermeiros o momento que se deve efetuar a retriagem dos pacientes com base em alterações significativas nos sinais vitais ou no tempo máximo definido pelo protocolo para fazer a retiragem.

O protótipo desenvolvido utilizou uma ontologia do protocolo de Manchester, que é um dos mais utilizados no mundo e muito comum nas emergências de hospitais brasileiros, durante o processo de avaliação. Apesar do protocolo de Manchester possuir mais de cinquenta fluxogramas [Mackway-Jones et al. 2014], que devem ser utilizados de acordo com a apresentação de cada paciente, na avalição proposta foi utilizado apenas o fluxograma para Asma e os discriminadores voltados a esse fluxograma. Sendo assim, após a triagem do paciente, o serviço começa a escutar modificações nos sinais vitais enviadas pela interface do paciente e alerta o enfermeiro responsável pela triagem quando, no caso de pacientes com asma, houverem alterações significativas na temperatura corporal, pulsação, espirometria ou na oximetria de pulso do paciente. Segundo o protocolo de Manchester, alguns outros discriminadores também afetam a triagem do paciente, como o nível de consciência do paciente e a capacidade de falar pequenas sentenças sem a necessidade de respirar [Mackway-Jones et al. 2014]. Entretanto, esses discriminadores não foram utilizados na avaliação proposta.

$\mathrm{O}$ aplicativo que faz interface com o paciente e enfermeiro foi desenvolvido para a plataforma de dispositivos móveis iOS. As telas desse protótipo podem ser observadas na Figura 3, que apresenta as visões do (A) enfermeiro e do (B) paciente. Sua construção utilizou a linguagem nativa para desenvolvimento nessa plataforma, denominada ObjectiveC. O aplicativo que faz interface com o paciente fica conectado ao servidor enviando todas as alterações nos sinais vitais do paciente. Sendo assim, sempre que for identificada alguma mudança significativa, o enfermeiro responsável pela triagem do paciente é

\footnotetext{
${ }^{1}$ Disponível em http://symfony.com.

${ }^{2}$ Dispoível em http://www.doctrine-project.org.
} 
notificado para que efetue a retriagem.
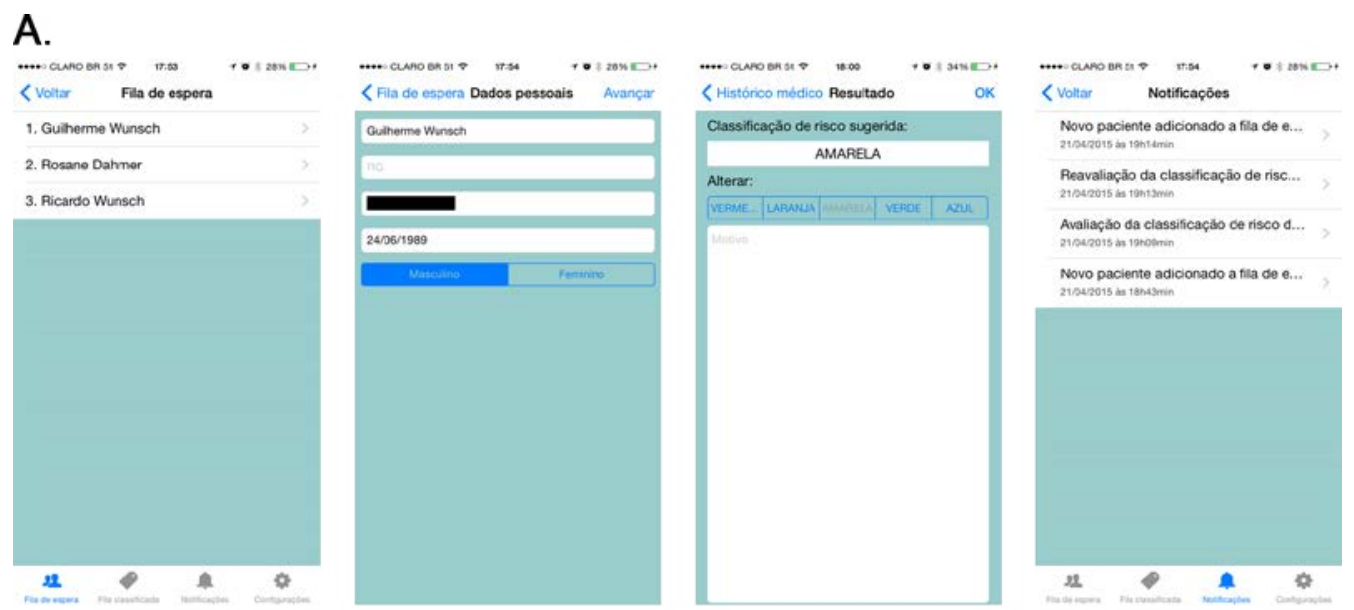

B.
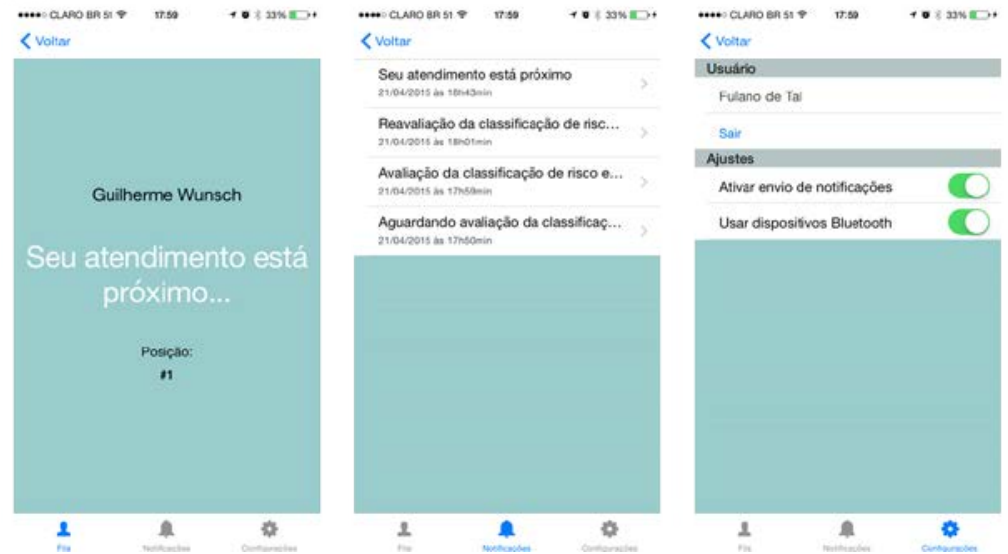

Figura 3. Telas do protótipo desenvolvido: Interface dos (A) enfermeiros e dos (B) pacientes.

No cenário criado para a avaliação do modelo, a paciente inicialmente foi triada com a cor laranja, entretanto durante a avalição as medições de oximetria de pulso e da pulsação do paciente alteraram. Essas alterações foram enviadas ao serviço que detectou uma retriagem do paciente. Nesse mesmo momento o enfermeiro responsável recebeu uma notificação para que fosse efetuada essa retriagem. Para que esse resultado fosse alcançado, foram desenvolvidos, também na linguagem Objective-C, simuladores dos sensores citados anteriormente, que foram acoplados ao módulo responsável pela integração com dispositivos vestíveis para monitoramento dos sinais vitais. Esse módulo não foi desenvolvido por completo, pois foi o meio encontrado para conseguir chegar mais próximo da situação criada no cenário proposto para a avaliação.

Com isso, foi possível verificar que o modelo se comportou de maneira adequada, garantindo que o a retriagem da paciente fosse efetuada em seu devido momento, ou seja, quando, através da ciência de situação, foi detectado que as informações de sinais vitais, aferidas através de sensores vestíveis, sofreram alterações significativas. Sendo assim, o protótipo confirmou que o modelo foi capaz de detectar, de forma ubíqua, alterações no quadro dos pacientes notificando enfermeiros quando fosse necessário efetuar a retriagem. 


\section{Trabalhos relacionados}

Alguns modelos, que se propõem a monitorar os sinais vitais de pacientes, podem ser encontrados na literatura. Entretanto, esses modelos não estão direcionados diretamente ao processo de triagem de pacientes nas emergências de hospitais. Dentre os modelos encontrados, foram escolhidos os mais representativos em relação ao modelo proposto.

O trabalho de [Borodin et al. 2015] se propõe a desenvolver uma arquitetura, utilizando o conceito de Internet das coisas, para um sistema de monitoramento de saúde usando diferentes fontes para monitorar dados voltados à saúde. O modelo prevê suporte a eletrocardiograma, batimentos cardíacos e outros, como, espirômetro, estetoscópio e tonômetro. A arquitetura ainda define o uso de serviços na nuvem para armazenar e tratar registros remotamente.

Já [Hassanalieragh et al. 2015] se preocupa em definir como deve ser uma arquitetura para monitoramento de saúde usando Internet das coisas e processamento em nuvem. O modelo desenvolvido trata desde a aquisição de dados voltados à saúde dos usuários, através de sensores conectados a um concentrador (que pode estar localizado em um smartphone), até a transmissão desses dados de forma segura e modularizada para posterior armazenamento e análise com uma interface de visualização intuitiva que facilita a leitura dos dados.

Uma arquitetura com uma camada de hardware que suporta múltiplos protocolos para conectar dispositivos clínicos, sensores de ambiente e sistemas de diferentes fontes de dados foi proposta por [Jara et al. 2012]. Ela transmite de forma contínua todos os dados coletados para uma outra camada, já em software, que é responsável por modularizar e proteger esses dados para serem transmitidos, em tempo real, à uma plataforma capaz de monitorar, de forma proativa, os dados recebidos. Gerando alertas e possibilitando sua utilização para o diagnóstico remoto.

Por fim, o modelo proposto em [da Rocha et al. 2015], permite o monitoramento da saúde utilizando dispositivos conectados através de uma rede Bluetooth ao smartphone do paciente. Esse modelo analisa o contexto do paciente, com base nos sinais fisiológicos recebidos e envia alterações da sua saúde a um serviço na nuvem capaz de efetuar o processamento de situação para tomar ações de acordo com a classificação de risco definida pela análise efetuada previamente. Essas ações vão desde enviar orientações ao paciente, até notificar o seu cuidador e, posteriormente, um centro de telemedicina.

Comparando os trabalhos relacionados, podemos observar que nenhum dos modelos propostos faz uso de informações pertinentes a saúde dos pacientes para auxiliar no processo de triagem . Outra lacuna que foi considerada muito importante, e, também, não explorada pelos modelos mencionados, é retriagem de pacientes. A Tabela 1 facilita a compreensão dessa comparação e apresenta as principais características dos trabalhos relacionados, incluindo o modelo proposto pelo presente trabalho.

\section{Conclusão}

O presente trabalho teve como objetivo elaborar um modelo computacional para auxiliar os enfermeiros no processo de retriagem em emergências de hospitais usando os conceitos da computação móvel e ubíqua e Internet das coisas. A crescente ascensão dos dispositivos móveis e a necessidade de maior agilidade e menor subjetividade nas funções 
Tabela 1. Comparação de características dos trabalhos relacionados em relação ao modelo proposto.

\begin{tabular}{c|c|c|c|c|c}
\hline Característica & [Borodin et al. 2015] & [Hassanalieragh et al. 2015] & [Jara et al. 2012] & [da Rocha et al. 2015] & Modelo proposto \\
\hline Uso de dispositivos móveis & Sim & Sim & Não & Sim & Sim \\
\hline Uso de sensores & Sim & Sim & Sim & Sim & Sim \\
\hline Serviço na nuvem & Sim & Sim & Não & Sim & Sim \\
\hline Análise de contexto & Não & Sim & Não & Sim & Sim \\
\hline Análise de situação & Não & Não & Não & Sim & Sim \\
\hline Uso na triagem de pacientes & Não & Não & Não & Não & Sim \\
\hline Uso na retriagem & Não & Não & Não & Não & Sim \\
\hline
\end{tabular}

desempenhadas pelos profissionais da área de saúde foram as principais motivações que ocasionaram na elaboração desse modelo. Sua principal contribuição científica, sendo uma das principais lacunas encontradas nos trabalhos relacionados, foi a avaliação da necessidade de efetuar a etapa de retriagem, em momento apropriado, a partir de dados de saúde coletados diretamente dos pacientes com o uso de Internet das coisas.

Para o processo de avaliação foi desenvolvido um protótipo cuja finalidade foi aplicá-lo a um cenário desenvolvido para validar o uso do modelo proposto. Com isso, foi possível verificar que o uso de ciência de situação se adéqua com eficiência ao processo de triagem, notificando os enfermeiros o momento em que se deve efetuar a retriagem de um paciente. Esse resultado foi possível ser alcançado com o uso de dispositivos vestíveis que monitoram os sinais vitais do paciente. Sendo assim, também é possível concluir que o uso da Internet das coisas agregou funcionalidades essenciais ao modelo desenvolvido e que, o modelo proposto é viável para utilização em emergências de hospitais.

Existem algumas oportunidades de trabalhos futuros relacionados a esse modelo. Inicialmente pode-se desenvolver as integrações com dispositivos vestíveis reais para o monitoramento de sinais vitais dos pacientes. Além disso, também é possível realizar um estudo de caso com pacientes em um ambiente controlado para analisar como a solução se comportaria em situações reais e, com isso, avaliar atributos como a usabilidade e percepção de valor desse modelo.

\section{Agradecimentos}

Os autores gostariam de agradecer a CAPES, ao CNPq e a FAPERGS pelo apoio a essa pesquisa.

\section{Referências}

Atzori, L., Iera, A., and Morabito, G. (2010). The internet of things: A survey. Computer networks, 54(15):2787-2805.

Blake, M. B. (2015). An internet of things for healthcare. IEEE Internet Computing, (4):4-6.

Borodin, A., Zavyalova, Y., Zaharov, A., and Yamushev, I. (2015). Architectural approach to the multisource health monitoring application design. In Open Innovations Association (FRUCT), 2015 17TH Conference of, pages 16-21. IEEE.

Christ, M., Grossmann, F., Winter, D., Bingisser, R., and Platz, E. (2010). Modern triage in the emergency department. Deutsches Ärzteblatt international, 107(50):892.

da Rocha, C. C. L., da Costa, C. A., and da Rosa Righi, R. (2015). Um modelo para monitoramento de sinais vitais do coração baseado em ciência da situação e computação ubíqua. In VII Simpósio Brasileiro de Computação Ubíqua e Pervasiva. 
Gelogo, Y. E. and Kim, H.-K. (2013). Unified ubiquitous healthcare system architecture with collaborative model. International Journal of Multimedia and Ubiquitous Engineering, 8(3).

Hassanalieragh, M., Page, A., Soyata, T., Sharma, G., Aktas, M., Mateos, G., Kantarci, B., and Andreescu, S. (2015). Health monitoring and management using internet-ofthings (iot) sensing with cloud-based processing: Opportunities and challenges. In Services Computing (SCC), 2015 IEEE International Conference on, pages 285-292. IEEE.

Jara, A. J., Zamora, M. A., and Skarmeta, A. F. (2012). Knowledge acquisition and management architecture for mobile and personal health environments based on the internet of things. In Trust, Security and Privacy in Computing and Communications (TrustCom), 2012 IEEE 11th International Conference on, pages 1811-1818. IEEE.

Jentsch, M., Ramirez, L., Wood, L., and Elmasllari, E. (2013). The reconfiguration of triage by introduction of technology. In Proceedings of the 15th international conference on Human-computer interaction with mobile devices and services, pages 55-64. ACM.

Jobé, J., Ghuysen, A., Gérard, P., Hartstein, G., and D’Orio, V. (2014). Reliability and validity of a new french-language triage algorithm: the elisa scale. Emergency Medicine Journal, 31(2):115-120.

Krafzig, D., Banke, K., and Slama, D. (2005). Enterprise SOA: service-oriented architecture best practices. Prentice Hall Professional.

Mackway-Jones, K., Marsden, J., and Windle, J. (2014). Emergency triage. Blackwell Publishing, 3 edition.

Marilza Pernas, A., Diaz, A., Motz, R., and Palazzo Moreira de Oliveira, J. (2012). Enriching adaptation in e-learning systems through a situation-aware ontology network. Interactive Technology and Smart Education, 9(2):60-73.

Rahmat, M. H., Annamalai, M., Halim, S. A., and Ahmad, R. (2013). Agent-based modelling and simulation of emergency department re-triage. In Business Engineering and Industrial Applications Colloquium (BEIAC), 2013 IEEE, pages 219-224. IEEE.

Satyanarayanan, M. (2011). Mobile computing: the next decade. ACM SIGMOBILE Mobile Computing and Communications Review, 15(2):2-10.

Schilit, B., Adams, N., and Want, R. (1994). Context-aware computing applications. In First Workshop on Mobile Computing Systems and Applications, 1994. WMCSA 1994, pages 85-90. IEEE.

Weiser, M. (1991). The computer for the 21st century. Scientific american, 265(3):94104.

Westergren, H., Ferm, M., and Häggström, P. (2014). First evaluation of the paediatric version of the swedish rapid emergency triage and treatment system shows good reliability. Acta Paediatrica, 103(3):305-308.

Worster, A., Sardo, A., Eva, K., Fernandes, C., and Upadhye, S. (2007). Triage tool inter-rater reliability: a comparison of live versus paper case scenarios. Journal of emergency nursing, 33(4):319-323. 